\title{
Prevalência de Salmonella sp. em suínos abatidos no Estado de Mato Grosso
}

\author{
Prevalence of Salmonella sp. in swine slaughtered at Mato Grosso state, Brazil \\ Maria Cristina da SilvaI Givago Silva FariaII Daphine Ariadne Jesus de Paula ${ }^{\text {II }}$ \\ Rodrigo Prado Martins ${ }^{\mathrm{II}}$ João Garcia Caramori Junior ${ }^{\mathrm{III}}$ Jalusa Deon Kich ${ }^{\mathrm{IV}}$ \\ Edson Moleta Colodel ${ }^{\mathrm{II}}$ Luciano Nakazato ${ }^{\mathrm{II}}$ Valéria Dutra ${ }^{\mathrm{II}}$
}

\section{RESUMO}

O presente trabalho teve como objetivo determinar a prevalência de Salmonella sp. em suínos abatidos em frigoríficos sob Inspeção Federal no Estado de Mato Grosso. Amostras de linfonodos mesentéricos e tonsilas de diferentes procedências foram coletadas em três frigoríficos. Das 300 amostras processadas, 50 (16,6\%) foram positivas para Salmonella sp., sendo identificados 14 diferentes sorovares. Os sorovares mais freqüentes foram Derby (16\%), Typhimurium (14\%), London (12\%) e Give (12\%). Os resultados epidemiológicos obtidos mostram uma baixa prevalência desta bactéria quando comparados com os resultados de estudos realizados em outros Estados.
\end{abstract}

- NOTA-
Palavras-chave: Salmonella, suínos, Mato Grosso.

\section{ABSTRACT}

The present study aims to determine the prevalence of Salmonella sp. in pigs in slaughterhouses under Federal Inspection at Mato Grosso, Brazil. Samples of mesenteric lymph nodes and tonsils were collected in three abattoirs from different regions. Three hundred samples were processed; fifty (16.6\%) were positive for Salmonella sp. and belonged to fourteen different serological types. The most common serotypes were Derby (16\%), Typhimurium (14\%), London (12\%) and Give (12\%). The epidemiological results obtained show a low prevalence of this bacterium when compared to studies in other Brazilian states.

Key words: Salmonella, swine, Mato Grosso.
No Brasil, a situação dos rebanhos e dos produtos de origem suína em relação à presença de Salmonella sp. é pouco conhecida. A prevalência da bactéria em suínos ao abate foi determinada no Rio Grande do Sul (BESSA et al., 2004) e em São Paulo (TEIXEIRA, 2006), com índices de 55,6\% e 23,91\%, respectivamente. No entanto, informações em relação à infecção pela bactéria em rebanhos de outros Estados são escassos. Portanto, o objetivo deste trabalho foi determinar a prevalência de Salmonella sp. em suínos abatidos em frigoríficos sob Inspeção Federal no Estado de Mato Grosso (MT) e fornecer dados epidemiológicos regionais para orientação e implementação de programas de controle.

A amostragem para a determinação da prevalência de suínos positivos ao abate foi calculada pelo programa EPI-Info (DEAN et al., 2007), sendo fornecidos os seguintes parâmetros: número de animais abatidos sob Inspeção Federal no Estado (800.000 animais ano ${ }^{-1}$ ), prevalência estimada $10 \%$, intervalo de confiança de $95 \%$ e precisão absoluta de $10 \%$. A coleta das amostras, na primeira carcaça, foi estabelecida ao acaso e as demais foram escolhidas em um intervalo calculado a partir do número previsto de animais abatidos dividido pelo número de amostras a serem coletadas.

\footnotetext{
IPrograma de Pós-graduação em Ciência Animal, Universidade Federal do Mato Grosso (UFMT), Cuiabá, MT, Brasil.

IIDepartamento de Cínica Médica Veterinária, UFMT, Avenida Fernando s/nº Coxipó, 78060-900, Cuiabá, MT, Brasil. E-mail: valdutra@ufmt.br.*Autor para correspondência.

IIIDepartamento de Ciências Básicas e Produção Animal, UFMT, Cuiabá, MT, Brasil.

IVEmbrapa-CNPSA, Concórdia, SC, Brasil.
} 
Foram coletadas 300 amostras (196 de linfonodos mesentéricos e 104 de tonsilas de diferentes animais) entre junho de 2005 e julho de 2007, oriundas de 13 municípios do estado do MT. As amostras foram identificadas, acondicionadas em coletor plástico estéril individual, mantidas e transportadas sob refrigeração até o processamento. Para o isolamento, amostras de $10 \mathrm{~g}$ de linfonodos mesentéricos e tonsilas foram processadas conforme metodologia adaptada de MICHAEL et al. (2003). As amostras foram submetidas às etapas de pré-enriquecimento em água peptonada tamponada $1 \%$ e enriquecimento em meios seletivos Rappaport Vassiliadis e Tetrationato e semeadura em agar XLT4 e verde brilhante. As colônias suspeitas foram submetidas a provas bioquímicas de triagem, conforme metodologia proposta por QUINN et al. (1993), sendo que as amostras positivas bioquimicamente foram encaminhadas à Fundação Instituto Oswaldo Cruz (FIOCRUZ-RJ) para sorotipificação.

Das 300 amostras analisadas, encontrou-se uma prevalência de 16,6\% de Salmonella sp. em suínos ao abate. Deste total, 12 (11,54\%) corresponderam a tonsilas e 38 (19,39\%) a linfonodos mesentéricos. Este percentual é o mais baixo encontrado no país. Resultados mais próximos foram observados por TEIXEIRA (2006) no Estado de São Paulo, com prevalência de 23,91\%. Prevalências mais altas foram descritas em suínos abatidos na região Sul do país, com índices variando de 55,56\% a 77\% (BESSA et al., 2004; SCHWARZ, 2006). Alguns estudos têm apontado que suínos positivos em linfonodos mesentéricos são portadores assintomáticos de infecção prévia ocorrida na granja. Devido ao estresse do transporte até o abatedouro, esses animais poderiam excretar Salmonella sp. nas fezes, contaminando outros suínos, que passariam a ser positivos no conteúdo intestinal e nas tonsilas (MORROW et al., 2000). Apesar de o baixo índice de prevalência encontrado nas tonsilas, este achado representa um risco maior, pois, em conjunto com os músculos da região da cabeça, elas são utilizadas na fabricação de embutidos e, caso não ocorra tratamento térmico na indústria, estes chegarão contaminados ao consumidor (BERENDS et al., 1996; CASTAGNA et al., 2004a).

A baixa prevalência encontrada nos lotes avaliados pode estar associada a múltiplos fatores. $\mathrm{O}$ estado do Mato Grosso possui, principalmente, sistema de produção em ciclo completo, o que possivelmente interfere nas condições sanitárias do rebanho. Nesse sistema, os suínos são submetidos a condições menos estressantes, pois não ocorre a mistura de lotes de diferentes propriedades durante as fases de produção (LETELLIER et al., 2001).
Outros fatores seriam as condições de transporte e abate no estado de MT, onde o número de animais abatidos por lote ainda é menor em relação a outros estados. Estudos comprovaram a influência do tempo de permanência em currais pré-abate com alto nível de contaminação residual por Salmonella sp. sobre o número de animais positivos ao abate (ROSTAGNO, 2001; HURD et al., 2002).

A sorotipificação das amostras isoladas mostrou uma grande diversidade, sendo identificados 14 diferentes sorotipos. A sorotipificação não foi realizada em uma amostra pela ausência de Salmonella sp. e sete amostras não foram analisadas (Tabela 1). Os sorovares mais prevalentes foram Derby (16\%), Typhimurium (14\%), London (12\%) e Give (12\%). A ocorrência de mais de um sorovar foi observada em sete propriedades. Esta diversidade de sorovares já foi relatada em uma mesma granja por MICHAEL et al. (2002) e CASTAGNA et al. (2004a).

O sorotipo Derby também foi o mais prevalente no estudo realizado por NEIVA (1946). Em estudos realizados no Sul do país, os sorovares mais freqüentemente encontrados foram Typhimurium, Derby, Agona, Bredeney e Panama (WEISS et al., 2002; BESSA et al., 2004; CASTAGNA et al., 2004a; SCHAWRZ et al., 2007). A diversidade dos sorotipos pode estar relacionada a diferentes fontes de contaminação originadas na granja, no frigorífico ou por contaminação cruzada durante o abate (FUNK \& GEBREYES, 2004; KICH et al., 2005).

Tabela 1 - Freqüência de sorotipos de Salmonella isolados de suínos abatidos no Estado do MT entre junho de 2005 e julho de 2007.

\begin{tabular}{lc}
\hline Sorotipos & № isolado (\%) \\
\hline Derby & $8(16)$ \\
Typhimurium & $7(14)$ \\
Give & $6(12)$ \\
London & $6(12)$ \\
Agona & $5(10)$ \\
Rissen & $2(4)$ \\
Hadar & $1(2)$ \\
Enterica & $1(2)$ \\
Cubana & $1(2)$ \\
Infantis & $1(2)$ \\
Bredeney & $1(2)$ \\
Orion & $1(2)$ \\
Panama & $1(2)$ \\
Rugosa & $1(2)$ \\
Ausência & $1(2)$ \\
Não analisadas & $7(14)$ \\
Total & $50(100)$ \\
\hline
\end{tabular}


Alguns dos sorotipos identificados no presente estudo têm sido descritos em suínos portadores, rações e produtos de origem suína no Brasil (FIALHO et al., 1985; BESSA et al., 2004; CASTAGNA et al., 2004b; SCHWARZ et al., 2007). Os sorotipos que não causam doença clínica nos suínos são os de maior importância para segurança alimentar, uma vez que os animais não apresentam sinais clínicos, mas são fontes permanentes de contaminação desde a granja até o consumidor (BERENDS et al., 1996).

Os resultados obtidos demonstram uma baixa prevalência desta bactéria comparada a outros Estados, devido provavelmente a fatores ambientais relacionados ao sistema de criação e às condições de abate. Estudos futuros deverão ser realizados para avaliar os fatores de risco envolvidos na infecção.

\section{AGRADECIMENTOS}

À Fundação Oswaldo Cruz (FIOCRUZ), pela realização da sorotipagem das amostras, e à Fundação de Amparo à Pesquisa do Estado de Mato Grosso (FAPEMAT), pelo apoio financeiro.

\section{REFERÊNCIAS}

BERENDS, B.R. et al. Identification and quantification of risk factors in animal management and transport regarding Salmonella spp. in pigs. International Journal Food Microbiology, v.30 p.37-53, 1996.

BESSA, M.C. et al. Prevalência de Salmonella sp. em suínos abatidos em frigoríficos do Rio Grande do Sul. Pesquisa Veterinária Brasileira, v.24, p.80-84, 2004.

CASTAGNA, S.M.F. et al. Presença de Salmonella sp. no trato intestinal e em tonsilas/linfonodos submandibulares de suinos ao abate. Arquivo Brasileiro de Medicina Veterinaria e Zootecnia, v.56, p.300-306, 2004a.

CASTAGNA, S.M.F. et al. Prevalência de suínos portadores de Salmonella sp ao abate e contaminação de embutidos tipo frescal. Acta Scientiae Veterinariae, v.32, p.141-147, 2004b.

DEAN A.G. et al. Epi Info ${ }^{\mathrm{TM}}$, a database and statistics program for public health professionals. Atlanta, Georgia, USA: Centers for Disease Control and Prevention., 2007. Acessado em 20 mar. 2008. Online. Disponível na internet http://www.cdc.gov/epiinfo/.

FIALHO, E.T. et al. Composição química e ocorrência de Salmonella em alimentos e concentrados utilizados em ração de suínos. Pesquisa Agropecuária Brasileira, v.20, p.377384, 1985.

FUNK, J.A.; GEBREYES, W.A. Risk factor associated with Salmonella prevalence on swine farms. Journal of Swine Health and Production, v.12, p.246-251, 2004.
HURD, H.S. et al. Salmonella enterica infections in Market swine with and without transport and holding. Applied and Environmental Microbiology, v.68, p.2375-2381, 2002.

KICH, J.D. et al. Fatores associados à soroprevalência de Salmonella em rebanhos comerciais de suínos. Ciência Rural, v.35, n.2, p.398-405, 2005.

LETELLIER, A. et al. Host response to various treatments to recuce Salmonella infections in swine. Canadian Journal Veterinary Research. v.65, p.168-172, 2001.

MICHAEL, G.B. et al. Comparison of different selective enrichment steps to isolated Salmonella sp. from feces of Finishing Swine. Journal of Microbiology, v.34, p.138-142, 2003.

MICHAEL, G.B. et al. Sorotipos de Salmonella isolados em uma propriedade de suinos de terminação no sul do Brasil. Ciência Rural, v.32, p.525-527, 2002.

MORROW, W.E.M. et al. The prevalence of Salmonella spp. in feces on farm and in ceca at slaughter. In: PROCEEDINGS OF INTERNATIONAL PIG VETERINARY SOCIETY CONGRESS, 16., 2000, Melbourne. Anais... Melbourne: IPVS, 2000. p.207.

NEIVA, C. Incidência de salmonellas em suínos. Memorias Instituto Butantan, v.7, p.430-435, 1946.

QUINN, P.J. et al. Clinical veterinary microbiology. London: Wolfe, 1993. p.127-136.

ROSTAGNO, M. Infecção por Salmonella spp em suínos durante o descanso pré-abate. In: CONGRESSO BRASILEIRO DE VETERINÁRIOS ESPECIALISTAS EM SUÍNOS, 10., 2001, Porto Alegre. Anais... Concórdia: Embrapa suínos e aves, 2001. V.1,. p.119-120.

SCHWARZ, P. et al. Estudo longitudinal da Infecção por Salmonella enterica em rebanho suíno no Sul do Brasil. In: CONGRESSO BRASILEIRO DE VETERINARIOS ESPECIALISTAS EM SUÍNOS, 13., 2007, Florianópolis, Anais... Concórdia : Embrapa suínos e aves , 2007. V.1, p.291-293.

SCHWARZ, P. Prevalência sorológica e de isolamento de Salmonella enterica em suínos abatidos no sul do Brasil. 2006. 47f. Dissertação (Mestrado Ciências Veterinárias ) Programa de Pós-graduação em Ciências Veterinárias, Universidade Federal do Rio Grande do Sul, Porto Alegre.

TEIXEIRA, R.S. Detecção de Salmonella spp. em amostras de fezes, linfonodos e carcaças de suínos no momento do abate. 2006. 50f. Dissertação (Mestrado Medicina Veterinária ) - Programa de Pós-graduação em Epidemiologia Experimental e Aplicada às Zoonoses, Universidade de São Paulo, SP.

WEISS, L.H.N. et al. Ocorrência de Salmonella spp. em suínos de terminação no Rio Grande do Sul. Pesquisa Veterinária Brasileira, v.22, p.104-108, 2002. 